\title{
Probabilistic Conformance for Cyber-Physical Systems
}

\author{
Yu Wang \\ yu.wang094@duke.edu \\ Duke University \\ Durham, NC \\ Borzoo Bonakdarpoor \\ borzoo@msu.edu \\ Michigan State University \\ East Lansing, MI
}

\author{
Mojtaba Zarei \\ mojtaba.zarei@duke.edu \\ Duke University \\ Durham, NC \\ Miroslav Pajic \\ miroslav.pajic@duke.edu \\ Duke University \\ Durham, NC
}

\begin{abstract}
In system analysis, conformance indicates that two systems simultaneously satisfy the same set of specifications of interest; thus, the results from analyzing one system automatically transfer to the other, or one system can safely replace the other in practice. In this work, we study the probabilistic conformance of cyber-physical systems (CPS). We propose a notion of (approximate) probabilistic conformance for sets of complex specifications expressed by the Signal Temporal Logic (STL). Based on a novel statistical test, we develop the first statistical verification methods for the probabilistic conformance of a wide class of CPS. Using this method, we verify the conformance of the startup time of the widely-used full and simplified model of Toyota powertrain systems, the settling time of model-predictive-control-based and neural-network-based automotive lane-keeping controllers, as well as the maximal voltage deviation of full and simplified power grid systems.
\end{abstract}

\section{ACM Reference Format:}

Yu Wang, Mojtaba Zarei, Borzoo Bonakdarpoor, and Miroslav Pajic. 2021. Probabilistic Conformance for Cyber-Physical Systems. In ACM/IEEE 12th International Conference on Cyber-Physical Systems (with CPS-IoT Week 2021) (ICCPS '21), May 19-21, 2021, Nashville, TN, USA. ACM, New York, NY, USA, 12 pages. https://doi.org/10.1145/3450267.3450534

\section{INTRODUCTION}

Conformance is an important concept in the analysis of cyberphysical systems (CPS) [13, 18, 21, 23, 32, 34]. It indicates that two systems satisfy the same set of given specifications (e.g., reachability or input-output relation). Thus the analysis results for one system can transfer to the other system, or one system can safely replace the other in practice. The term "conformance" may also refer to the consistency between a system and a design specification (e.g., $[14,22])$; this is out of the scope of this work.

For CPS, complex specifications for their dynamics are mathematically expressible by temporal logics, such as the Signal Temporal Logic (STL) [24]. Following the line of work [1,9], we focus on the conformance of CPS for temporal logics specifications. This

Permission to make digital or hard copies of part or all of this work for personal or classroom use is granted without fee provided that copies are not made or distributed for profit or commercial advantage and that copies bear this notice and the full citation on the first page. Copyrights for third-party components of this work must be honored

For all other uses, contact the owner/author(s).

ICCPS '21, May 19-21, 2021, Nashville, TN, USA

(c) 2021 Copyright held by the owner/author(s).

ACM ISBN 978-1-4503-8353-0/21/05.

https://doi.org/10.1145/3450267.3450534 notion of conformance generalizes the conformance for reachability $[21,32]$, since reachability is expressible by temporal logic.

Conformance can be used for two different models derived from the same system under two conditions, implying that the system executes in the same way under the conditions (e.g., two inputs). A well-known example of nonconformity is the Volkswagen emissions scandal [4], where the emission control software deliberately performs differently in the lab testing and driving conditions to bypass the emission test without actually reducing the pollution generated from the cars while driving. Similar undesirable nonconformity exists in printers [5], where the software drivers deliberately work differently in favor of certain cartridge brands. To prevent such software doping [31], one needs to verify the conformance of a system under different conditions/settings.

The conformance also applies to two models derived from two systems operating under the same conditions, implying that they are interchangeable for the application. For instance, there has been recently significant interest in replacing precise but computationally expensive controllers based on model predictive control (MPC) with ones based on neural network $(\mathrm{NN})$ for applications such as lane-keeping systems in autonomous vehicles [29]. To migrate from an MPC controller to an NN controller without significantly changing the responsiveness, we need to check the conformance of the closed-loop system under the two controllers for their settling time, especially considering the fragility of AI-based controllers. While we focus on the conformance of two different systems operating under the same conditions in our case studies, our approach also applies to a system's conformance under two conditions.

Since CPS, such as autonomous vehicles, are frequently subject to randomness (e.g., system/network/environment noise), we propose a probabilistic notion of conformance for these systems. We use the definition of probabilistic uncertain systems (PUSs) from [40] to capture CPS dynamics. Roughly speaking, they are grey-box probabilistic dynamical systems with unknown dynamics in known state space. The PUSs capture the system nondeterminism as the input and probabilism as the parameters. The input and parameters can be time functions of general types, including real, integer, or categorical/Boolean. Given the input and parameters' value, a timedependent sample path of general types can be generated. The PUSs subsume commonly used dynamical models such as continuoustime Markov chains and hybrid I/O automata [15] with probabilistic parameters (used to capture the Toyota Powertrain [33]).

We define the notion of conformance through a parameterized signal temporal logic (STL) formula [3] as illustrated in Figure 1. 


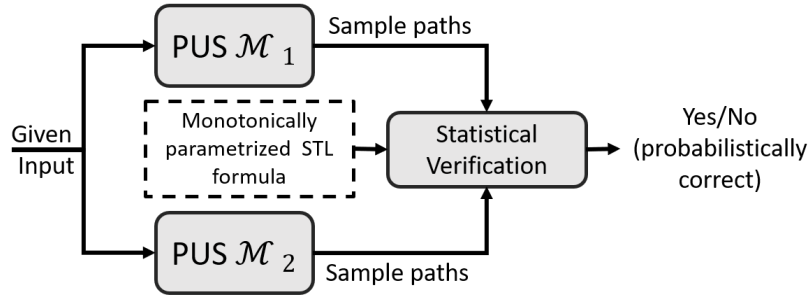

Figure 1: Overview of our statistical conformance test.

Specifically, we require that the satisfaction probabilities are approximately equal for all values of the STL parameters. For example, for the probabilistic conformance of two models $\mathcal{M}_{1}$ and $\mathcal{M}_{2}$ of reaching the same set $\mathcal{D}$, one can consider the parameterized formula $\diamond_{[0, t]} \mathcal{D}$ and require that for a given $c>0$, it holds that

$$
\begin{aligned}
\forall t \in[0, \infty) . & \mid \operatorname{Pr}_{\sigma_{1} \sim \mathcal{M}_{1}}\left(\sigma_{1} \vDash \diamond_{[0, t]} \mathcal{D}\right) \\
& -\operatorname{Pr}_{\sigma_{2} \sim \mathcal{M}_{2}}\left(\sigma_{2}=\diamond_{[0, t]} \mathcal{D}\right) \mid<c ;
\end{aligned}
$$

here, $\sigma_{1}$ and $\sigma_{2}$ are two random signals from the models $\mathcal{M}_{1}$ and $\mathcal{M}_{2}$, respectively, as illustrated in Figure 2. This implies that both systems $\mathcal{M}_{1}$ and $\mathcal{M}_{2}$ reach $\mathcal{D}$ with approximately equal probability for any time horizon. Our notion of conformance only requires these probabilities to be approximately equal instead of exactly equal, since the former is usually sufficient in practice (more examples are provided in Section 6).

Since the PUSs may have complex or even unknown dynamics, we adopt a statistical verification approach, as it scales better than model-based verification approaches and can handle unknown dynamics [2, 19]. From the conformance definition, we need to simultaneously handle the approximately equal satisfaction probability of infinitely many STL specifications since the parameters of the parameterized STL formula can take infinitely many values; this is very challenging since existing statistical verification methods can only handle a single (non-parametrized) temporal logic formula $[2,20]$ or a hyper temporal logic formula $[38,40]$.

We show that statistically verifying conformance is feasible when the STL formula is monotonically parameterized, i.e., the formula's satisfaction probability changes monotonically with the parameters. Such a property holds for many cases as discussed in detail in Section 3 and the case studies in Section 6. To the best of our knowledge, this work is the first to enable statistical verification for infinitely many formulas.

Due to monotonicity, the satisfaction probabilities over the values of its parameters on the two PUSs form two probability distributions. Accordingly, the conformance of two PUSs requires the two distributions to be approximately equal. To this end, we develop a new statistical test to check the approximate equality of two distributions with provable confidence levels. Our test is based on the classic Kolmogorov-Smirnov (KS) test [10] and its multivariate generalization [30] for checking the exact equality of two distributions. Based on this, we develop a statistical verification method for the probabilistic (non)conformance of two PUSs for any desired confidence level (lower than 1).

We apply the proposed statistical verification method to check the probabilistic conformance for three case studies to show its

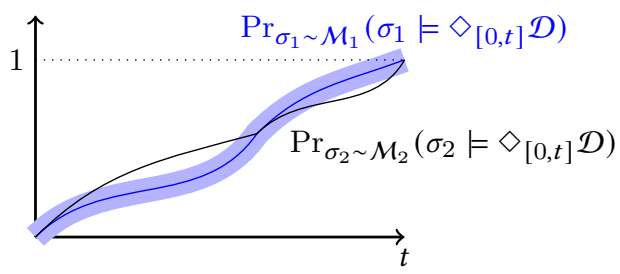

Figure 2: Reachability probabilities for some set $\mathcal{D}$ v.s. time horizon $t$. The two models conform (for reachability) if the black line stays within the blue tube.

applicability. First, we study the probabilistic conformance of the widely used full and simplified models of the Toyota powertrain system [16, 33] for the startup time for their air to fuel ratio to reach a working region. Our results show the nonconformity of the two models, suggesting the simplified model may not capture certain important aspects of the system. Second, we check the probabilistic conformance of the settling time of an MPC-based lanekeeping controller and several NN-based lane-keeping controllers of different sizes for an autonomous car [26]. We show that NNbased controllers conform to the MPC-based controller, as their size increases; however, a small NN design may result in nonconformity. It suggests that an MPC-based controller can be replaced with a sufficiently-large NN-based controller to satisfyingly control the settling time. Finally, we check the probabilistic conformance of the maximal deviation of DC voltage between the full model and a simplified model of a power grid system [27]. Our results show that the two models do not probabilistically conform - i.e., the simplified model again may not capture certain important aspects of the system.

This paper is organized as follows. After preliminaries in Section 2, in Section 3 we formalize the problem and our definition of probabilistic conformance for a parameterized STL formula. We present a new statistical test in Section 4 and the verification method for the probabilistic conformance in Section 5. In Section 6, we apply our method to three real-world case-studies, before discussing related work in Section 7, and concluding in Section 8.

Notation. We denote the sets of natural, real, and non-negative real numbers by $\mathbb{N}, \mathbb{R}$, and $\mathbb{R}_{\geq 0}$, respectively. We define $\mathbb{R}_{\infty}=$ $\mathbb{R} \cup\{-\infty, \infty\}$, and $[n]=\{1, \ldots, n\}$, for $n \in \mathbb{N}$. The cardinality and the power set of a set $S$ are denoted by $|S|$ and $2^{S}$.

\section{PROBLEM FORMULATION}

We use a general system model for CPS called probabilistic uncertain systems (PUSs) [40]. They capture continuous-time probabilistic dynamics on a hybrid state-space of discrete and continuous values, as well as generalize common probabilistic models such as continuous-time Markov chains (CTMC) and probabilistic hybrid I/O automata [40]. Since we adopt a statistical approach, we mainly view a PUS as a grey-box that generates random samples (Figure 3).

DeFINITION 1. A probabilistic uncertain system (PUS) is a tuple $\mathcal{M}=\left(\mathcal{X}, X_{\text {init }}, \mathcal{I}, \mathcal{D},\{D(t)\}_{t \in \mathbb{R}_{\geq 0}}, \mathcal{T}\right)$, where

- $\mathcal{X}=\mathcal{X}_{1} \times \ldots \times \mathcal{X}_{n}$ is the state space with each $\mathcal{X}_{i}$ being either $\mathbb{R}$ or a discrete set $\left[n_{X_{i}}\right]$; 


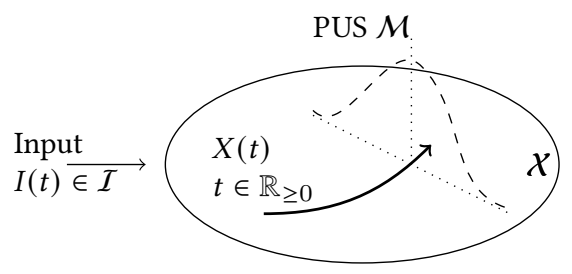

Figure 3: Probabilistic Uncertain System (PUS).

- $X_{\text {init }} \in \mathcal{X}$ is the initial state;

- $\mathcal{I}=\mathcal{I}_{1} \times \ldots \times \mathcal{I}_{m}$ is the range of inputs with each $\mathcal{I}_{i}$ being either $\mathbb{R}$ or a discrete set $\left[n_{\mathcal{I}_{i}}\right]$;

- $\mathcal{D}=\mathcal{D}_{1} \times \ldots \times \mathcal{D}_{l}$ is the range of parameters with each $\mathcal{D}_{i}$ being either $\mathbb{R}$ or a discrete set $\left[n_{\mathcal{D}_{i}}\right]$;

- $\{D(t)\}_{t \in \mathbb{R}_{\geq 0}}$ is a random process on $\mathcal{D}$ (for a properly defined probability space), defining the random change of the parameter over time;

- $\mathcal{T}:\left(\mathbb{R}_{\geq 0} \rightarrow \mathcal{I}\right) \times\left(\mathbb{R}_{\geq 0} \rightarrow \mathcal{D}\right) \rightarrow\left(\mathbb{R}_{\geq 0} \rightarrow \mathcal{X}\right)$ defines the transition of the system - i.e., given the (time-dependent) value of the input and parameter, the system deterministically generates a path.

Given the value of the (time-dependent) input $I: \mathbb{R}_{\geq 0} \rightarrow \mathcal{I}$, the PUS can generate a random signal $\sigma(t)=\mathcal{T}(I(t), D(t))$, where the randomness comes from the parameter $D(t)$. We denote by $\sigma \sim \mathcal{M}_{I}$ when the signal $\sigma$ is randomly generated from the system $\mathcal{M}$ for the given input $I$. We also write $\sigma \sim \mathcal{M}$ if $I$ is clear from the context.

There is no assumption on the dynamics of a PUS, such as Markovian, causal, etc. Common probabilistic models such as the discrete-time or continuous-time Markov chains [36], and probabilistic hybrid I/O automata $[35,42]$ are subsumed by the notion of PUS (see [40] for details).

EXAMPLE 1. A simple example of PUS is a bouncing ball with random gravitational acceleration, as shown in Figure 4. Its state is the height and velocity $(x, v)$. For $x>0$, the state evolves by $\dot{x}=v, \dot{v}=g$; for $x=0$, it jumps by $x \mapsto x, v \mapsto-v$. The parameter $g$ is randomly drawn from a normal distribution $N\left(g_{0}, \sigma^{2}\right)$ for some $g_{0}, \sigma>0$. The initial state is $\left(x_{0}, 0\right)$. The input set is empty.

Finally, note that although by Definition 1, a PUS has a unique initial state, it allows for defining conformance of paths from different initial states $X_{1}$ and $X_{2}$ of the PUS. This is done by adding a new initial state $X_{0}$ to the PUS, and model the transition from $X_{0}$ to $X_{1}$ and $X_{2}$ as part of the input.

Signal Temporal Logic. We use the signal temporal logic (STL) [24] to capture the temporal specifications of interest for the random signals of the PUS. STL can be viewed as the counterpart of linear temporal logic (LTL) in the real-time domain with real-valued constraints. An STL formula is defined inductively by the syntax

$$
\varphi::=f>0|\neg \varphi| \varphi \wedge \varphi \mid \varphi \mathcal{U}_{\left[t_{1}, t_{2}\right]} \varphi,
$$

where $f: \mathbb{R}^{n} \rightarrow \mathbb{R}$ is a given function. To simplify further discussion, we let $t_{1}, t_{2} \in \mathbb{R}_{\infty}$, instead of taking values in nonnegative rational numbers. We call $f>0$ an atomic proposition and $\mathcal{U}_{\left[t_{1}, t_{2}\right]}$ the "until" operator. Other temporal and logic operators are defined as usual; for example,

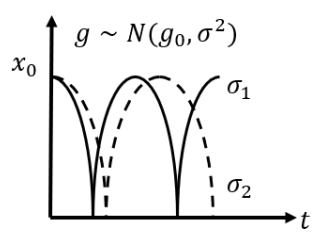

Figure 4: Stochastic bouncing ball.

- (false/true) $\mathrm{F}=\varphi \wedge(\neg \varphi)$ and $\mathrm{T}=\neg \mathrm{F}$,

- (finally) $\diamond_{\left[t_{1}, t_{2}\right]} \varphi=\mathrm{T} \mathcal{U}_{\left[t_{1}, t_{2}\right]} \varphi$, and

- (always) $\square_{\left[t_{1}, t_{2}\right]} \varphi=\neg\left(\diamond\left[t_{1}, t_{2}\right] \neg \varphi\right)$.

For a concrete signal $\sigma: \mathbb{R}_{\geq 0} \rightarrow \mathbb{R}^{n}$ of the PUS, the satisfaction relation for STL formulas is defined recursively by the semantics

$$
\begin{aligned}
& \sigma \mid=f>0 \quad \text { iff } f(\sigma(0))>0 \\
& \sigma \mid=\neg \varphi \quad \text { iff } \sigma \not \models \varphi \\
& \sigma=\varphi_{1} \wedge \varphi_{2} \quad \text { iff } \sigma \mid=\varphi_{1} \text { and } \sigma \models \varphi_{2} \\
& \sigma=\varphi_{1} \mathcal{U}_{\left[t_{1}, t_{2}\right]} \varphi_{2} \text { iff there exists } t \in\left[t_{1}, t_{2}\right] \text { such that } \\
& \sigma^{(t)} \vDash \varphi_{2} \text { and for any } 0 \leq t^{\prime}<t \text {, } \\
& \text { it holds that } \sigma^{\left(t^{\prime}\right)}=\varphi_{1} \text {; }
\end{aligned}
$$

here, $\sigma^{(t)}$ denotes the $t$-shift of the signal, defined by $\sigma^{(t)}\left(t^{\prime}\right)=$ $\sigma\left(t+t^{\prime}\right)$ for any $t^{\prime} \in \mathbb{R}_{\geq 0}$.

We make the convention that a formula $\varphi_{1} \mathcal{U}_{\left[t_{1}, t_{2}\right]} \varphi_{2}$ is equivalent to $\mathrm{F}$, if $t_{2}<t_{1}, t_{1}<0$, or $t_{2}<0$.

EXAMPLE 2. The following STL formula requires that if $|x|>0.5$, then within 0.6 time units $|x|$ settles under the value 0.5 for the 1.5-long time interval

$$
\varphi=\square\left(|x|>0.5 \Rightarrow \diamond_{[0, .6]}\left(\square_{[0,1.5]}|x|<0.5\right)\right) .
$$

\section{PROBABILISTIC CONFORMANCE}

We focus on a class of conformance properties for CPS for an (infinite) set of STL formulas. Mathematically, we say that two PUSs probabilistically conform if for any STL formula from the set, the satisfaction probabilities are approximately equal for two random signals drawn respectively from the two PUSs. This can be viewed as a probabilistic generalization of $[1,9]$.

Definition 2 (CONFormance). Let $\Phi$ be an infinite set of STL formulas. For two PUSs $\mathcal{M}_{1}$ and $\mathcal{M}_{2}$, and a given $c>0$, we say that $\mathcal{M}_{1}$ and $\mathcal{M}_{2}$ c-approximately probabilistically conform for $\Phi$ (for the same given input), if for any STL formula $\phi \in \Phi$, it holds that

$$
\left|\operatorname{Pr}_{\sigma_{1} \sim \mathcal{M}_{1}}\left(\sigma_{1} \vDash \phi\right)-\operatorname{Pr}_{\sigma_{2} \sim \mathcal{M}_{2}}\left(\sigma_{2} \vDash \phi\right)\right|<c,
$$

where $\sigma_{i} \sim \mathcal{M}_{i}$ is a random path from the PUS $\mathcal{M}_{i}$, for $i \in\{1,2\}$.

In Definition 2, we only require the satisfaction probabilities to be approximately equal for the STL formulas of interest instead of exactly equal; the latter is usually unnecessary in applications (see e.g. the case studies presented in Section 6). Besides, the conformance from Definition 2 cannot be expressed by single formulas in any common temporal logic since a parameterized formula effectively captures an infinite number of STL formulas. For any fixed values of the employed parameters, the property can be expressed in HyperPSTL [40]. 
Depending on the choice of the class (i.e., set) of temporal properties $\Phi$, different notions for the conformance of PUS are derived, including probabilistic reach-set conformance and probabilistic trace conformance. Commonly, an STL formula set $\Phi$ can be derived by parametrizing a single STL formula $\phi$ by [3]

$$
\Phi=\left\{\phi_{\underline{d}}: \underline{d} \in \mathbb{R}^{K}\right\} .
$$

Effectively, $\phi_{\underline{d}}$ represents infinitely many STL formulas, as the parameter $\underline{d}$ can take infinitely many values.

For example, the STL formula set

$$
\Phi_{1}=\left\{\diamond_{[0,1]}(\sigma>a): a \in \mathbb{R}\right\}
$$

is derived by parametrizing the threshold $a$. It contains an infinite set of reachability specifications for the parametrized threshold $a$ within the fixed time-interval $[0,1]$. The conformance of the two PUSs $\mathcal{M}_{1}$ and $\mathcal{M}_{2}$ for the set $\Phi_{1}$ means that, for any threshold $a$ the probability of reaching the threshold should be approximately equal for two random signals respectively from $\mathcal{M}_{1}$ and $\mathcal{M}_{2}$.

Similarly, the STL formula set

$$
\Phi_{2}=\left\{\diamond_{[0, t]}(\sigma>0): t \in \mathbb{R}\right\}
$$

is derived by parametrizing the time horizon $t$. It contains an infinite set of reachability specifications for the fixed threshold 0 , within a parameterized time interval $[0, t]$. The conformance of the two PUSs $\mathcal{M}_{1}$ and $\mathcal{M}_{2}$ for the set $\Phi_{2}$ means that the probability of reaching the threshold 0 (i.e., $>0$ ) within any time interval $[0, t]$ should be approximately equal for two random signals respectively from $\mathcal{M}_{1}$ and $\mathcal{M}_{2}$.

Considering that the PUSs can have complex dynamics that may be even unknown in practice, in this work we propose to statistically verify the conformance of PUSs from Definition 2; such method exhibits better scalability than the exhaustive approaches and can handle unknown dynamics [2, 19]. There are infinitely many STL formulas of interest in (2), so the proposed statistical verification method should be able to handle an infinite set of STL specifications. This is very challenging since all existing statistical verification techniques can only handle single STL specifications $[2,20]$. To solve this, we focus on the conformance for monotonically parameterized STL formulas, which are commonly used in system analysis [3].

Generally, the parametrized formula $\phi_{d}$ (where $\underline{d}$ captures the vector of parameters) is monotone if the satisfaction probability on a model is preserved for the order of the parameters - i.e., the satisfaction probability changes monotonically with the parameter. While statistically verifying the probabilistic conformance for an arbitrary STL formula set is very difficult, handling a monotonically parameterized formula set can be done by exploiting the formula's monotonicity.

Definition 3 (Monotonically Parameterized Formula). $A$ parameterized formula $\phi_{\underline{d}}$ with $\underline{d} \in \mathbb{R}^{K}$ is monotone for a PUS $\mathcal{M}$ if for any given path $\sigma$ from $\mathcal{M}$ and $i \in[K]$, and

- for any $\underline{d}, \underline{d^{\prime}}$ such that $\underline{d} \unlhd_{i} \underline{d^{\prime}}$, it holds that $\sigma=\phi_{\underline{d}}$ implies $\sigma=\phi_{\underline{d^{\prime}}}, O R$

- for any $\underline{d}, \underline{d^{\prime}}$ such that $\underline{d} \leq_{i} \underline{d^{\prime}}$, it holds that $\sigma=\phi_{\underline{d^{\prime}}}$ implies $\sigma=\phi_{\underline{d}} ;$ here, $\underline{d} \leq_{i} \underline{d^{\prime}}$ denotes that the entries of $\underline{d}$ and $\underline{d^{\prime}}$ are equal except for $\underline{d}_{i} \leq{\underline{d^{\prime}}}_{i}$.

Following Definition 3, the parameter alternation preserves the parametrized STL formula's monotonicity.

Definition 4 (Alternation). The function $\pi(\underline{d})=\underline{d}^{\prime}$ is called an alternation, if for all $i \in[K], d_{i}^{\prime}=d_{i}$ or $d_{i}^{\prime}=-\bar{d}_{i}$. The set of all $K$-dimensional alternations in $\mathbb{R}^{K}$ is denoted by $\Pi_{K}$.

From the previous definitions, the following directly holds.

LEMMA 1. If $\phi_{\underline{d}}$ is a monotonically parameterized STL formula, then so is $\phi_{\pi(\underline{d})}$, where $\pi$ is an alternation. ${ }^{1}$

\section{STATISTICAL TEST FOR APPROXIMATE EQUALITY OF DISTRIBUTIONS}

Before introducing a statistical verification algorithm for probabilistic conformance, we propose a new statistical test for the equivalence of two (unknown) probability distributions, based on the classic Kolmogorov-Smirnov test [10, 30]. We start from the scalar case and then extend to the multidimensional case.

Consider two $K$-dimensional random vectors $\underline{X}=\left(X_{1}, \ldots, X_{K}\right)$ and $\underline{Y}=\left(Y_{1}, \ldots, Y_{K}\right)$. For each $K$-dimensional alternation $\pi \in \Pi_{K}$, we define

$$
\begin{aligned}
& F^{\pi}(\underline{a})=\operatorname{Pr}_{\underline{X}}\left(\pi(\underline{X})_{1} \leq \pi(\underline{a})_{1}, \ldots, \pi(\underline{X})_{K} \leq \pi(\underline{a})_{K}\right), \\
& G^{\pi}(\underline{a})=\operatorname{Pr}_{\underline{Y}}\left(\pi(\underline{Y})_{1} \leq \pi(\underline{a})_{1}, \ldots, \pi(\underline{Y})_{K} \leq \pi(\underline{a})_{K}\right),
\end{aligned}
$$

where $\pi(\underline{X})_{i}$ is the $i^{t h}$ entry of $\pi(\underline{X})$, and the probabilities $\operatorname{Pr}_{X}$ and $\operatorname{Pr}_{Y}$ are taken for the random vectors $\underline{X}$ and $\underline{Y}$, respectively. If $\pi$ is the identity map, then $F^{\pi}$ and $G^{\pi}$ are respectively the cumulative distribution functions (CDFs) of $\underline{X}$ and $\underline{Y}$, which we denote by $F$ and $G$ to simplify our notation. Otherwise, $F^{\pi}$ and $G^{\pi}$ are the complimentary CDFs of $\underline{X}$ and $\underline{Y}$.

To measure the difference between the probability distributions of $\underline{X}$ and $\underline{Y}$, let

$$
\gamma_{\underline{X}, \underline{Y}}=\max _{\pi \in \Pi_{K}}\left\|F^{\pi}-G^{\pi}\right\|_{\infty},
$$

with $\|\cdot\|_{\infty}$ standing for the $L_{\infty}$ function norm. If $\gamma_{X}, \underline{Y}=0$, then $\underline{X}$ and $\underline{Y}$ have the same probability distributions.

The approximate equality of the probability distributions of $\underline{X}$ and $\underline{Y}$ is formulated as the hypothesis testing problem

$$
\mathcal{H}_{0}: \gamma_{\underline{X}, \underline{Y}}<c \quad \mathcal{H}_{1}: \gamma_{\underline{X}, \underline{Y}}>c,
$$

where $c>0$ is the given parameter for approximate equality. The alternation $\pi$ in (6) is necessary since two different multidimensional probability distributions may have the same CDFs but different complimentary CDFs.

Assumption 1. Similar to previous work on statistical verification [40,41], we assume $\gamma_{X}, \underline{Y} \neq c$, which ensures that as the number of samples increases, the samples will increasingly concentrate to support either $\mathcal{H}_{0}$ or $\mathcal{H}_{1}$ by the central limit theorem. Therefore, a statistical analysis based on the majority of the samples has increasing accuracy. This assumption is weaker than the "indifference region" adopted in other works on statistical verification [2, 20].

\footnotetext{
${ }^{1}$ The alternation of time horizon parameters in $\mathcal{U}$ (and other temporal operators) follows the aforementioned convention that $\varphi_{1} \mathcal{U}_{\left[t_{1}, t_{2}\right]} \varphi_{2}$ is equivalent to $\mathrm{F}$, if $t_{2}<t_{1}, t_{1}<0$, or $t_{2}<0$.
} 
REMARK 1. The hypothesis testing problem (7) cannot be handled by the classic Kolmogorov-Smirnov (KS) test [10] and its multivariate generalization [30], since they can only check for the exact equality of two probability distributions, i.e., the hypothesis testing problem

$$
\mathcal{H}_{0}^{\prime}: \gamma_{\underline{X}, \underline{Y}}=0 \quad \mathcal{H}_{1}^{\prime}: \gamma_{\underline{X}, \underline{Y}}>0 .
$$

To solve problem (7), we build on the KS test and introduce a new statistical test for any given confidence level $\alpha$ (i.e., the lowest probability that the test's assertion agrees with the truth in all cases). ${ }^{2}$ To facilitate presentation, we start from the scalar case and then move to the vector case.

\subsection{Scalar Random Variables}

If $X$ and $Y$ are scalar, ${ }^{3}$ then from (7), we have that $\gamma_{X, Y}=\|F-G\|_{\infty}$, where $F$ and $G$ are the CDFs of $X$ and $Y$, respectively. ${ }^{4}$ Given two sets of independent and identically distributed (i.i.d.) samples

$$
X^{[n]}=\left(X^{(1)}, \ldots, X^{(n)}\right), \quad Y^{[m]}=\left(Y^{(1)}, \ldots, Y^{(m)}\right),
$$

drawn respectively from $X$ and $Y$, the ECDFs of the samples are

$$
\begin{aligned}
& F_{X^{[n]}}(x)=\frac{1}{n} \sum_{i=1}^{n} \mathrm{I}\left(X^{(i)} \leq x\right), \\
& G_{Y^{[m]}}(y)=\frac{1}{m} \sum_{i=1}^{m} \mathrm{I}\left(Y^{(i)} \leq y\right),
\end{aligned}
$$

where $\mathrm{I}(\cdot)$ is the indicator function. Intuitively, the different $\gamma_{X, Y}$ can be statistically estimated by (as illustrated in Figure 5)

$$
\delta_{X^{[n]}, Y^{[m]}}=\left\|F_{X^{[n]}}-G_{Y^{[m]}}\right\|_{\infty} .
$$

When the numbers of samples $n, m \rightarrow \infty$, the ECDFs converges to the CDFs: $F_{X^{[n]}} \rightarrow F$ and $G_{Y^{[m]}} \rightarrow G,{ }^{5}$ and thus, $\delta_{X^{[n]}, Y^{[m]}} \rightarrow$ $\gamma_{X, Y}$ by Glivenko-Cantelli theorem [37]. Therefore, for the hypothesis testing problem (7), we propose the statistics assertion

$$
\mathcal{A}\left(X^{[n]}, Y^{[m]}\right)= \begin{cases}\mathcal{H}_{0}, & \text { if } \delta_{X^{[n]}, Y^{[m]}}<c, \\ \mathcal{H}_{1}, & \text { if } \delta_{X^{[n]}, Y^{[m]}}>c .\end{cases}
$$

For random samples $X^{[n]}$ and $Y^{[m]}$, the probability $\alpha$ that the assertion (11) agrees with the correct answer to the hypothesis testing problem (7) is called the confidence level. It depends on the discrepancy between $\gamma_{X, Y}$ and $\delta_{X^{[n]}, Y^{[m]}}$, which is bounded by

$$
d_{X^{[n]}, Y^{[m]}}=\left\|\left(F_{X^{[n]}}-F\right)-\left(G_{Y^{[m]}}-G\right)\right\|_{\infty}
$$

due to the triangle inequality

$$
\left|\delta_{X^{[n]}, Y[m]}-\gamma_{X, Y}\right| \leq d_{X^{[n], Y}{ }^{[m]}} .
$$

When the numbers of samples $n, m \rightarrow \infty$, the discrepancy $d_{X^{[n]}, Y^{[m]}}$ $\rightarrow 0$ with probability 1 . However, the probability distribution of the rescaled discrepancy $d_{X^{[n]}, Y}^{[m]} \sqrt{m n /(m+n)}$ (for random samples $\left.X^{[n]}, Y^{[m]}\right)$ is asymptotically invariant of $n, m$ and is independent of the CDFs $F$ and $G$, as formally stated below.

\footnotetext{
${ }^{2}$ The confidence level $\alpha$ is minimum of the p-values of the two hypothesis. Accordingly, we refer to $1-\alpha$ as the significance level, which is the maximal of the false positive and the false negative rates.

${ }^{3}$ For this scalar case, to simplify our notation, we denote $\underline{X}$ and $\underline{Y}$ as $X$ and $Y$.

${ }^{4}$ This does not hold in general for multidimensional random variables.

${ }^{5}$ More precisely, this is convergence in distribution.
}

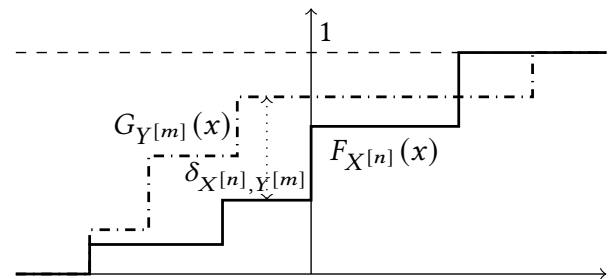

Figure 5: Illustration of the statistics $\delta_{X^{[n]}, Y^{[m]}}$.

Lemma 2 (Section 7.9 of [10]). The CDF $H(x)$ of the $d_{X^{[n]}, Y^{[m]}}$ $\sqrt{m n /(m+n)}$ from (12) obeys the Kolmogorov-Smirnov distribution

$$
H(x)=1-2 \sum_{i=1}^{\infty}(-1)^{i-1} e^{-2 i^{2} x^{2}} \approx 1-2 e^{-2 x^{2}} .
$$

Now, we derive the significance level (i.e., $1-\alpha$, where $\alpha$ is the confidence level ) of the assertion (11) when observing a value of the test statistics $\delta_{X^{[n]}, Y^{[m]}}$ to be $\lambda$. If $\lambda<c$, the significance level of (11) is the probability of observing a value of $\delta_{X^{[n]}, Y[m]}$ (in any other test) that is lower than $\lambda$ (i.e., more in favor of the hypothesis $\mathcal{H}_{0}$ ) under the likely-false hypothesis $\mathcal{H}_{1}$. It holds that ${ }^{6}$

$$
\begin{aligned}
1-\alpha & =\operatorname{Pr}_{X^{[n]}, Y^{[m]}}\left(\delta_{X^{[n]}, Y^{[m]}}<\lambda \mid \mathcal{H}_{1}\right) \\
& =\operatorname{Pr}_{X^{[n]}, Y^{[m]}}\left(\gamma_{X, Y}-\delta_{X^{[n]}, Y^{[m]}}>\gamma_{X, Y}-\lambda \mid \mathcal{H}_{1}\right) \\
& \leq \operatorname{Pr}_{X^{[n]}, Y^{[m]}}\left(d_{X^{[n]}, Y^{[m]}}>\gamma_{X, Y}-\lambda \mid \mathcal{H}_{1}\right) \\
& \leq \operatorname{Pr}_{X^{[n]}, Y^{[m]}}\left(d_{X^{[n]}, Y^{[m]}}>c-\lambda\right) \\
& =1-H((c-\lambda) \sqrt{m n /(m+n)})
\end{aligned}
$$

where (15) follows from (13); (16) holds since $\gamma_{X, Y}>c$ under $\mathcal{H}_{1}$; and (17) follows from Lemma 2. Similarly, if $\lambda>c$, the significance level of (11) satisfies

$$
1-\alpha \leq 1-H((\lambda-c) \sqrt{m n /(m+n)}) .
$$

Finally, combining (17) and (18), the confidence level of (11) satisfies

$$
\alpha \geq H(|\lambda-c| \sqrt{m n /(m+n)}) \text {. }
$$

Based on (19), for any desired confidence level $\alpha_{d}<1$, our statistical test is deployed sequentially. It can return an assertion with an actual confidence level of at least $\alpha_{d}$. Iteratively, the algorithm draws $k_{1}$ and $k_{2}$ new samples from the two CDFs $F$ and $G$, respectively, and then computes the actual confidence level $\alpha$ from (19). It terminates when $\alpha>\alpha_{d}$, and then returns the assertion by (11). This is formally captured in Algorithm 1.

Theorem 1. Algorithm 1 terminates with probability 1 and has the confidence level $\alpha_{d}$.

Proof. Termination: As $n, m \rightarrow \infty$, we have $\delta_{X^{[n]}, Y^{[m]}} \rightarrow$ $\gamma_{X, Y} \neq c$, - i.e., $\delta_{X^{[n]}, Y^{[m]}}$ converges to some value that is not $c$ with probability 1 , so either $\mathcal{H}_{0}$ or $\mathcal{H}_{1}$ holds. Therefore, Algorithm 1 terminates with probability 1 .

Correctness: Let $\tau$ be the step Algorithm 1 terminates and $A$ be "the assertion $\mathcal{A}$ from (11) is correct", then $\operatorname{Pr}(A)=\sum_{i \in \mathbb{N}} \operatorname{Pr}(A \mid \tau=$ i) $\operatorname{Pr}(\tau=i)$. From (19), for any $i \in \mathbb{N}$, we have that $\operatorname{Pr}(A \mid \tau=i)>$

\footnotetext{
${ }^{6}$ To simplify our notation, we also use $\delta_{X}[n]_{, Y}[m]$ to denote a random value of the test statistics in any other test in computing the significance level.
} 

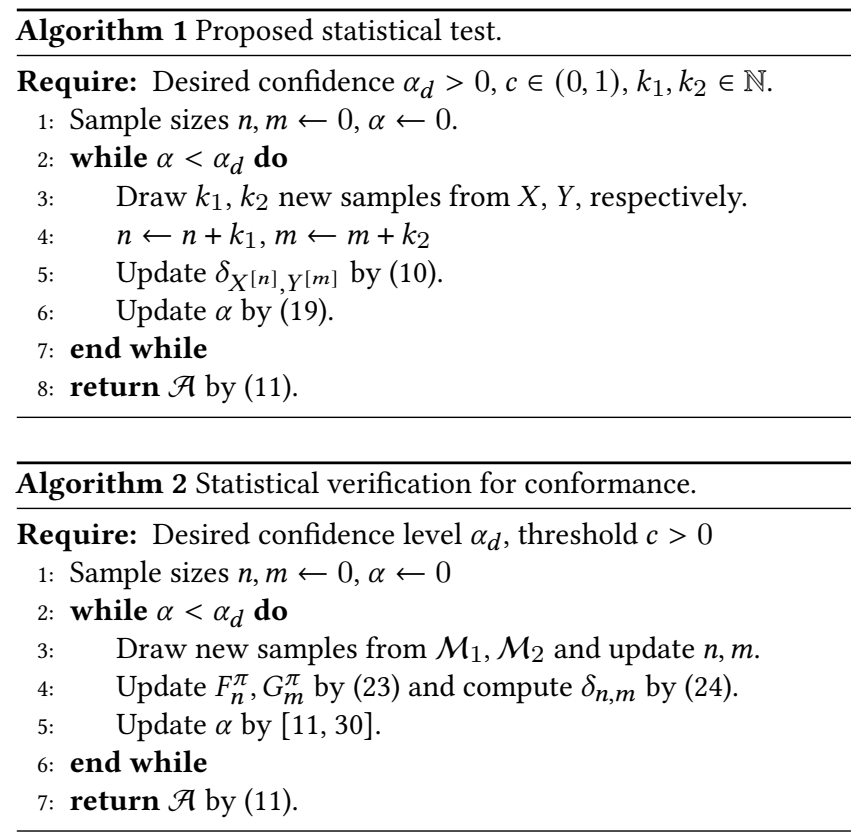

$\alpha_{d}$. In addition, by Termination, we have that $\sum_{i \in \mathbb{N}} \operatorname{Pr}(\tau=i)=1$, Therefore, it holds that $\operatorname{Pr}(A) \geq \alpha_{d}$.

REMARK 2. Although the test statistics $\delta_{X^{[n]}, Y^{[m]}}$ from (10) is also used in the standard KS test [10], the implementation and thresholding on $\delta_{X^{[n]}, Y^{[m]}}$ in (11) in our method fundamentally differs from the KS test. Specifically, our statistical test increasingly draws samples until reaching the desired confidence level $(<1)$ and the thresholding on $\delta_{X^{[n]}, Y}^{[m]}$ in (11) represents the similarity of the probability distributions as given in (7). On the other hand, the KS test employs a fixed number of samples and the thresholding on $\delta_{X^{[n]}, Y[m]}$ is related to the confidence level. Consequently, our method guarantees confidence levels for both $\mathcal{H}_{0}$ and $\mathcal{H}_{1}$ in (7), while the KS test only guarantees confidence level for $\mathcal{H}_{0}^{\prime}$, and not $\mathcal{H}_{1}^{\prime}$, in (8).

\subsection{Multidimensional Random Variables}

Similarly to the scalar case, for random vectors $\underline{X}$ and $\underline{Y}$, let $\underline{X}^{[n]}=$ $\left(\underline{X}^{(1)}, \ldots, \underline{X}^{(n)}\right)$ and $\underline{Y}^{[m]}=\left(\underline{Y}^{(1)}, \ldots, \underline{Y}^{(m)}\right)$ be two sets of i.i.d. samples from $\underline{X}$ and $\underline{Y}$, respectively. Then, we can define the ECDF and the complimentary ECDFs from $\underline{X}^{[n]}$ by

$$
F_{\underline{X}^{[n]}}^{\pi}(\underline{a})=\frac{1}{n} \sum_{i=1}^{n} \mathrm{I}\left(\pi\left(\underline{X}^{(i)}\right)_{1} \leq \pi(a)_{1}, \ldots, \pi\left(\underline{X}^{(i)}\right)_{K} \leq \pi(a)_{K}\right),
$$

for each $K$-dimensional alternation $\pi \in \Pi_{K}$ (given by Definition 4). Similarly, we can define $G_{Y^{[m]}}^{\pi}(\underline{a})$ from $\underline{Y}^{[m]}$.

Following [11, 30], we note that generally $\left\|F_{\underline{X}^{[n]}}^{\pi}-G_{\underline{Y}^{[m]}}^{\pi}\right\|_{\infty}$ are not equal for all $\pi \in \Pi_{K}$. Thus, defining the test statistics by only using the CDFs $F_{\underline{X}^{[n]}}^{\pi}$ and $G_{\underline{Y}^{[m]}}^{\pi}$ by $\delta_{X^{[n]}, Y^{[m]}}=\left\|F_{\underline{X}^{[n]}}-G_{\underline{Y}^{[m]}}\right\|_{\infty}$, as in (10) is not enough. Instead, the test statistics should take all the CDFs and complimentary CDFs by

$$
\delta_{X^{[n]}, Y^{[m]}}=\max _{\pi \in \Pi_{K}}\left\|F_{\underline{X}^{[n]}}^{\pi}-G_{\underline{Y}^{[m]}}^{\pi}\right\|_{\infty} .
$$

By $[11,30]$, the test statistics $\delta_{X^{[n]}, Y^{[m]}}$ satisfies Lemma 2 and asymptotically obeys the Kolmogorov-Smirnov distribution (14). Therefore, the statistical test (11) extends to the multidimensional case by using $\delta_{X^{[n]}, Y[m]}$ from (20). For $K \leq 3$, the confidence level for $(20)$ can be derived directly from the results of $[11,30]$. For $K>3$, it can be computed by extending the method of $[11,30]$.

\section{STATISTICAL VERIFICATION OF PROBABILISTIC CONFORMANCE}

Based on the statistical test introduced in Section 4, we now propose a statistical verification algorithm to check the probabilistic conformance of two PUSs for a monotonically parametrized STL formula (as formulated in Section 3). For a lucid presentation and as with most other works (e.g., $[2,19]$ ), we focus on bounded-time properties; handling unbounded-time properties is more involving and is an avenue for future work.

Following Definition 2, for a monotonically parametrized STL formula $\phi_{d}$ with $\underline{d} \in \mathbb{R}^{K}$ and for each $K$-dimensional alternation $\pi \in \Pi_{K}$ (from Definition 4), let

$$
\begin{aligned}
F^{\pi}(\underline{d}) & =\operatorname{Pr}_{\sigma_{1} \sim \mathcal{M}_{1}}\left(\sigma_{1} \vDash \phi_{\pi(\underline{d})}\right), \\
G^{\pi}(\underline{d}) & =\operatorname{Pr}_{\sigma_{2} \sim \mathcal{M}_{2}}\left(\sigma_{2} \vDash \phi_{\pi(\underline{d})}\right) .
\end{aligned}
$$

By the monotonicity of $\phi_{d}$ from Definition 3, for each for $\pi \in \Pi_{K}$, the multivariate functions $F^{\pi}$ is the CDF or a complementary CDF of the satisfaction probability of $\phi_{d}$ for the parameter $\underline{d}$. The equality in (21) is almost everywhere in Lebesgue measure, since distribution functions $F^{\pi}(\underline{d})$ and $G^{\pi}(\underline{d})$ need to be right-continuous. The same holds for $G^{\pi}$.

From Definition 2, the PUSs $\mathcal{M}_{1}$ and $\mathcal{M}_{2}$ conform with respect to the monotonically parametrized formula $\phi_{\underline{d}}$, if the CDFs and complementary $\mathrm{CDFs} F^{\pi}(\underline{d})$ and $G^{\pi}(\underline{d})$ are approximately equal; i.e.,

$$
\left|\operatorname{Pr}_{\sigma_{1} \sim \mathcal{M}_{1}}\left(\sigma_{1} \vDash \phi\right)-\operatorname{Pr}_{\sigma_{2} \sim \mathcal{M}_{2}}\left(\sigma_{2}=\phi\right)\right|<c
$$

if and only if

$$
\gamma_{\underline{X}, \underline{Y}}=\max _{\pi \in \Pi_{K}}\left\|F^{\pi}-G^{\pi}\right\|_{\infty}<c .
$$

On the other hand, this can be solved by our statistical test introduced in Section 4.

Specifically, for two sets of sample paths $\sigma_{1}^{[n]}=\left(\sigma_{1}^{(1)}, \ldots, \sigma_{1}^{(n)}\right)$ and $\sigma_{2}^{[m]}=\left(\sigma_{2}^{(1)}, \ldots, \sigma_{2}^{(m)}\right)$ from the PUSs $\mathcal{M}_{1}$ and $\mathcal{M}_{2}$, respectively, we define the empirical approximations of $F(\underline{d})$ and $G(\underline{d})$ by

$$
\begin{aligned}
F_{n}^{\pi}(\underline{d}) & =\frac{1}{n} \sum_{i=1}^{n} \mathrm{I}\left(\sigma_{1}^{(i)} \models \phi_{\pi(\underline{d})}\right), \\
G_{m}^{\pi}(\underline{d}) & =\frac{1}{m} \sum_{i=1}^{m} \mathrm{I}\left(\sigma_{2}^{(i)} \models \phi_{\pi(\underline{d})}\right),
\end{aligned}
$$

where $\pi \in \Pi_{K}$ and $\mathrm{I}(\cdot)$ is the indicator function. ${ }^{7}$ Similarly to (20), the test statistics

$$
\delta_{n, m}=\max _{\pi \in \Pi_{K}}\left\|F_{n}^{\pi}-G_{m}^{\pi}\right\|_{\infty},
$$

where $\delta_{n, m}$ is the $L_{\infty}$ norm, satisfies Lemma 2 and obeys the KS distribution from (14) (asymptotically for $K \geq 2$ ); hence, the statistical test (11) applies. Since $F_{n}^{\pi}$ and $G_{m}^{\pi}$ are known multidimensional step functions from the samples, $\left\|F_{n}^{\pi}-G_{m}^{\pi}\right\|_{\infty}$ is directly computable.

\footnotetext{
${ }^{7}$ In the rest of this paper, we use simplified notation with subscripts ()$_{n}$ and ()$_{m}$ utilized to indicate the sets of sample paths $\sigma_{1}^{[n]}$ and $\sigma_{2}^{[m]}$.
} 


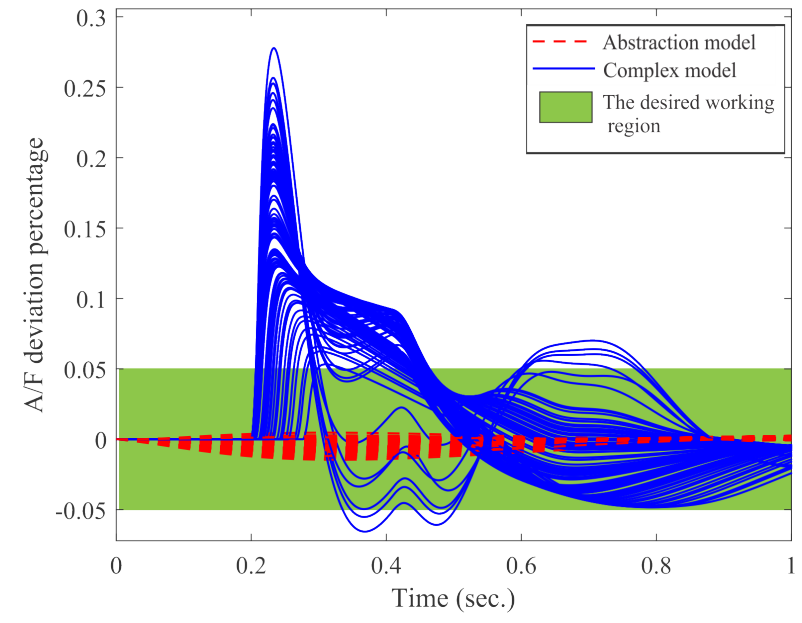

Figure 6: Sample paths from the complex (solid blue) and abstracted (dashed red) models for the $A / F$ ratio deviation percentage. The paths remain inside the desired working region (in green) with a significantly higher probability for the abstracted model, illustrating that the distribution of the arrival times to the desired working region is very different for these two models.

Algorithm 2 for checking probabilistic conformance terminates with probability 1 and can achieve any desired confidence level $\alpha_{d}<1$. The proof follows from that of Theorem 1 .

\section{CASE STUDIES AND EVALUATION}

To demonstrate the applicability of our statistical verification algorithms, we evaluated them on three CPS benchmarks with complex dynamics from a wide range of application domains: (1) Toyota Powertrain, (2) Lane-Keeping Assistant (LKA) Controllers, and (3) $100 \mathrm{~kW}$ Grid-Connected Photo Voltaic (PV) Array (due to space constraints the results of the $3^{r d}$ case study are presented in the Appendix). We find the case study in Section 6.2 particularly important since (LKA controllers), to the best of our knowledge, previously there are few comparative studies between NN-based and conventional techniques in cyber-physical and embedded systems.

The Toyota powertrain model is derived from [16]. The LKA is implemented in MATLAB using the MPC, Deep Learning, and Reinforcement Learning Toolboxes [25]. The PV Array is implemented using the Simscape Power Systems toolbox [28]. All implementations are available at [6].

Evaluations are performed on a laptop with Intel Xeon E-2176M CPU @ 2.7GHz and 16 GB RAM. For each case study, we run Algorithm 2 with different indifference parameter $c$ and desired confidence level $\alpha_{d}$ (i.e., the probability for Algorithm 2 to return the correct assertion is at least $\alpha_{d}$ ). We report the test statistics $\delta_{n, m}$, the number of samples, total algorithm execution time, and the assertion $\mathcal{A}$ when the algorithm terminates.

\subsection{Toyota Powertrain}

We use the Simulink models for the Toyota Powertrain with a four-mode embedded controller and 15 state variables from [16]. It is challenging to show that complex embedded/CPS with hybrid dynamics, such as the powertrain, satisfy strict performance requirements. On the one hand, the available benchmark model must capture a reasonable portion of behaviors of the real powertrain to enable us to assess, evaluate, and verify the designs against requirements. On the other hand, the simulation time for a simpler model that sufficiently conforms with the real system is significantly lower.

In [16], two models of the Toyota Powertrain are presented. A detailed but complex model contains the air-to-fuel (A/F) ratio controller and an average model of the engine dynamics, such as the throttle and intake manifold air dynamics. Due to the complexity of this detailed model and limitations of existing verification tools, in [16], a simpler abstract model as a hybrid I/O automaton is also introduced to facilitate system analysis, including formal verification.

Conformance. For the Toyota powertrain, the A/F ratio control problem is of key interest. Hence, we study the conformance for the $\mathrm{A} / \mathrm{F}$ deviations $e_{A / F}$ for the detailed and abstract models for an RPM of 1600 (the system input). When the nominal input RPM is subject to Gaussian system noise $\mathrm{N}\left(0,18^{2}\right)$, (samples of) the change of $e_{A / F}$ over time for the two models are given in Figure 6. The conformance requires that, under this system noise, the $\mathrm{A} / \mathrm{F}$ deviations $e_{A / F}$ of the detailed and abstract models enter some desired working region $\left(\left|e_{A / F}\right|<0.05\right)$ in any time interval $[0.22, \tau]$ with approximately the same probability; i.e., the STL specification $\diamond_{[0.22, \tau]}\left(\left|e_{A / F}\right|<\right.$ $0.05)$ holds with approximately the same probability for any $\tau$ between the two models, as formally captured below ${ }^{8}$

$$
\begin{aligned}
\forall \tau \geq 0 . & \operatorname{Pr}_{\sigma_{a} \sim \mathcal{M}_{a}}\left(\sigma_{a} \vDash \diamond_{[0.22, \tau]}\left(\left|e_{A / F}\right|<0.05\right)\right) \\
& \approx_{c} \operatorname{Pr}_{\sigma_{f} \sim \mathcal{M}_{f}}\left(\sigma_{f}=\diamond_{[0.22, \tau]}\left(\left|e_{A / F}\right|<0.05\right)\right) .
\end{aligned}
$$

Here, the constant $c>0$, the approximate equality $\approx_{c}$ means the difference is less than $c$, the subscripts $f$ and $a$ stand for the complex and abstracted models, respectively, $e_{A / F}$ is the percentage deviation of $\mathrm{A} / \mathrm{F}$ ratio, and $\tau$ is the time-bound.

Result Analysis. We analyzed (25) using Algorithm 2 with the confidence level $\alpha \in\{0.95,0.99\}$ and the conformance parameter $c \in\{0.2,0.15,0.10,0.05\}$ (see Table 1 ). The results are derived with relatively small numbers of samples for all confidence and indifference parameters. The results indicate that the two employed models do not conform for the requirement (25), although it is claimed in [16] that the abstract model is a representative of the detailed model. Starting from the same initial RPM values, the $\mathrm{A} / \mathrm{F}$ ratio in the complex model would take more time to reach the desired working region than in most cases in the abstracted model. This also agrees with Figure 6, as the A/F ratio of the abstracted model would remain inside the desired area, while in the complex model, this value exceeds the desired region in most of the cases. Furthermore, from Table 1, the value of the test statistics $\delta_{n, m}$ is almost 1 in all the cases, when Algorithm 2 terminates. This implies that for the detailed and abstracted models, the distribution of the startup time for their $\mathrm{A} / \mathrm{F}$ ratio to reach the working region are very different - this agrees with the algorithm assertion.

${ }^{8}$ More precisely, for any $\tau \geq 0.22$ from (25). Otherwise, the satisfaction probability is trivially 0 . 


\begin{tabular}{llllll}
$c$ & $\alpha_{d}$ & $\delta_{n, m}$ & Samples & Time (sec.) & $\mathcal{A}$ \\
\hline 0.40 & 0.99 & 1.00 & $3.9 \mathrm{e}+01$ & $1.8 \mathrm{e}-02$ & $\mathrm{~F}$ \\
0.40 & 0.95 & 1.00 & $1.9 \mathrm{e}+01$ & $4.4 \mathrm{e}-03$ & $\mathrm{~F}$ \\
0.25 & 0.99 & 1.00 & $2.5 \mathrm{e}+01$ & $4.6 \mathrm{e}-03$ & $\mathrm{~F}$ \\
0.25 & 0.95 & 1.00 & $1.3 \mathrm{e}+01$ & $2.2 \mathrm{e}-03$ & $\mathrm{~F}$ \\
0.10 & 0.99 & 1.00 & $1.8 \mathrm{e}+01$ & $3.6 \mathrm{e}-03$ & $\mathrm{~F}$ \\
0.10 & 0.95 & 1.00 & $9.0 \mathrm{e}+00$ & $1.6 \mathrm{e}-03$ & $\mathrm{~F}$ \\
0.05 & 0.99 & 1.00 & $1.6 \mathrm{e}+01$ & $2.8 \mathrm{e}-03$ & $\mathrm{~F}$ \\
0.05 & 0.95 & 1.00 & $8.0 \mathrm{e}+00$ & $1.3 \mathrm{e}-03$ & $\mathrm{~F}$
\end{tabular}

Table 1: Statistical verification results of the conformance property (25) and the test statistics $\delta_{n, m}$ upon Algorithm 2 termination for different values of conformance parameter $c$ and desired confidence level $\alpha_{d}$.

\subsection{Replacing MPC with NN-based Controllers}

The controller of the LKA system is commonly based on model predictive control (MPC) or more recently neural networks (NN). The conventional MPC-based controllers solve a constrained quadratic programming optimization problem from the observed state of a plant in an open-loop fashion. This approach is usually computationally ineffective in realtime. Recently, NN-based controllers are employed to imitate the control rules of the MPC-based controller from samples to improve realtime computation efficiency. In this case study, we check the conformance of an NN-based controller and an MPC-based controller for the LKA system in MATLAB/Simulink [25].

In the LKA system, the sensors measure the lateral deviation, relative yaw angle between the center-line of a lane and the vehicle, current lane curvature, and its derivative. The objective of the controller is to keep the lateral error and relative yaw angle close to zero. To dynamics of the vehicle is given by the three Degreesof-Freedom (DoF) bicycle model [12] as

$$
\begin{aligned}
{\left[\begin{array}{c}
\dot{V}_{y} \\
\dot{\psi}
\end{array}\right] } & =\left[\begin{array}{cc}
-\frac{2 C_{f}+2 C_{r}}{m V_{x}} & -V_{x}-\frac{2 C_{f} l_{f}-2 C_{r} l_{r}}{m V_{x}} \\
-\frac{2 C_{f} l_{f}-2 C_{r} l_{r}}{I_{z} V_{x}} & -\frac{2 C_{f} l_{f}^{2}+2 C_{r} l_{r}^{2}}{I_{z} V_{x}}
\end{array}\right]\left[\begin{array}{c}
V_{y} \\
\dot{\psi}
\end{array}\right]+2\left[\begin{array}{c}
\frac{C_{f}}{m} \\
\frac{C_{f}^{m} l_{f}}{I_{z}}
\end{array}\right] u \\
y & =\left[\begin{array}{ll}
V_{y} & \dot{\psi}
\end{array}\right]^{\mathrm{T}} .
\end{aligned}
$$

Here, $V_{x}$ is the longitudinal velocity, $m$ is the total vehicle mass, $I_{z}$ is the yaw moment of inertia of the vehicle, $l_{f}$ and $l_{r}$ are the longitudinal distance from the center of gravity to the front and real tires, and $C_{f}$ and $C_{r}$ are the cornering stiffness of the front and rear tires, respectively. The system state consist of the lateral velocity $V_{y}$ and yaw angle rate $\dot{\psi}$, and the front steering angle $u(t)$ is the system input.

MPC. The MPC-based controller is derived from the MPC toolbox in MATLAB. The values of the variables are set as follows: $V_{x}=15 \mathrm{~m} / \mathrm{s}, \mathrm{m}=1575 \mathrm{~kg}, I_{z}=2875 \mathrm{~m} \cdot \mathrm{N} \cdot \mathrm{s}^{2}, l_{f}=1.2 \mathrm{~m}$, $l_{r}=1.6 \mathrm{~m}, C_{f}=19000 \mathrm{~N} / \mathrm{rad}$, and $C_{r}=33000 \mathrm{~N} / \mathrm{rad}$. The controller output is confined within the interval $[-\pi / 3, \pi / 3] \mathrm{rad}$. The predictive time horizon and control time horizon are set to $h_{p}=20$ and $h_{c}=20$.

DNN Replacement. We train a NN controller to replace the MPC controller, by sampling from the MPC based controller for randomly generated states, last control action, and measured disturbances.
The samples are divided into the train and validation testing data, and are used to train several NNs with similar structure, but different numbers of neurons per layer $(30,45,60$, and 300 neurons per layer). All middle layers are fully connected with ReLU activation functions and the output layer is a fully-connected layer with tanh activation function and a scalar layer. The maximal number of epoch to stop the training is set to 30 . The structure of the NNs is shown in Figure 7.

Conformance. For the input of the same reference path of the vehicle (given by the Matlab Toolbox), we expect that using the $\mathrm{NN}$ controller the lateral deviation of the vehicle under random values of the initial states should be similar to the output of the MPC-based closed-loop system. Thus, we assign an upper bound to the error of the lateral deviation and check when the designed controller reaches this boundary. With fixed values of initial states, we run the closed-loop system with two NNs and the reference MPC. Then, we compare the time that the absolute value of the lateral deviation falls below the desired value for the NN controller and the MPC controller; this is formally captured by the STL formula $\diamond_{[0, \tau]}\left(\left|e_{y}\right|<\gamma\right)$ monotonically parametrized by $\tau$. Accordingly, the conformance between the MPC-controlled and NN-controlled LKA systems for this parametrized specification is

$$
\begin{aligned}
\forall \tau \geq 0 . & \operatorname{Pr}_{\sigma_{1} \sim \mathcal{M}_{\mathbf{N N}}}\left(\sigma_{1}=\diamond_{[0, \tau]}\left(\left|e_{y}^{\mathrm{NN}}\right|<\gamma\right)\right) \\
& \approx_{c} \operatorname{Pr}_{\sigma_{2} \sim \mathcal{M}_{\mathbf{M P C}}}\left(\sigma_{2} \mid=\diamond_{[0, \tau]}\left(\left|e_{y}^{\mathrm{MPC}}\right|<\gamma\right)\right),
\end{aligned}
$$

where the constants $c, \gamma>0$, the approximate equality $\approx_{c}$ means the difference is less than $c$, and $e_{y}$ is the lateral deviation of the intended controller. The random signals $\sigma_{1}$ and $\sigma_{2}$ are derived as follows. The initial conditions of the system such as the lateral velocity $V_{y}$, yaw angle rate $\dot{\psi}$, lateral deviation $e_{1}$, relative yaw angle $e_{2}$, last steering angle $u$, and the measured road yaw rate $V_{x} \rho$ are drawn randomly using the uniform distribution from intervals $[-2,2] \mathrm{m} / \mathrm{s},[-\pi / 3, \pi / 3] \mathrm{rad} / \mathrm{s},[-1,1] \mathrm{m},[-\pi / 4, \pi / 4] \mathrm{rad}$, $[-\pi / 3, \pi / 3] \mathrm{rad}$, and $[-0.01,0.01]$, respectively. The minimum road reduce is $100 \mathrm{~m}$.

Result Analysis. The results for applying Algorithm 2 with parameters $\alpha \in\{0.95,0.99\}$, and $c \in\{0.40,0.25,0.10,0.05\}$ are shown in Table 2 for NN controllers with 30 and 45 neurons per layer. As can be seen, the NN controllers with 45 neurons per layer conforms much better with the MPC controller than the NN controllers with 30 neurons per layer for the requirement (26). The results for 60 and 300 neurons per layer are similar to 45 neurons per layer (as confirmed by Figure 8), so they are omitted due to the space limit. All these results are achieved with relatively few samples (at most a few thousand samples for each setup).

The results of Table 2 imply that increasing the size of the NNbased controllers improves the conformance with the MPC controller. To check this observation and confirm the results of Table 2, we show in Figure 8 the ECDFs of the settling time for the MPC controller and the NN controllers with 30, 45, 60, and 300 neurons per layer; the conformance for the requirement (26) is visually demonstrated by the closeness of the ECDFs. To derive the same conclusion, each ECDF uses 200 samples, which is significantly more than the samples required by Algorithm 2, as shown in Table 2. As shown in Figure 8, increasing the number of neurons 


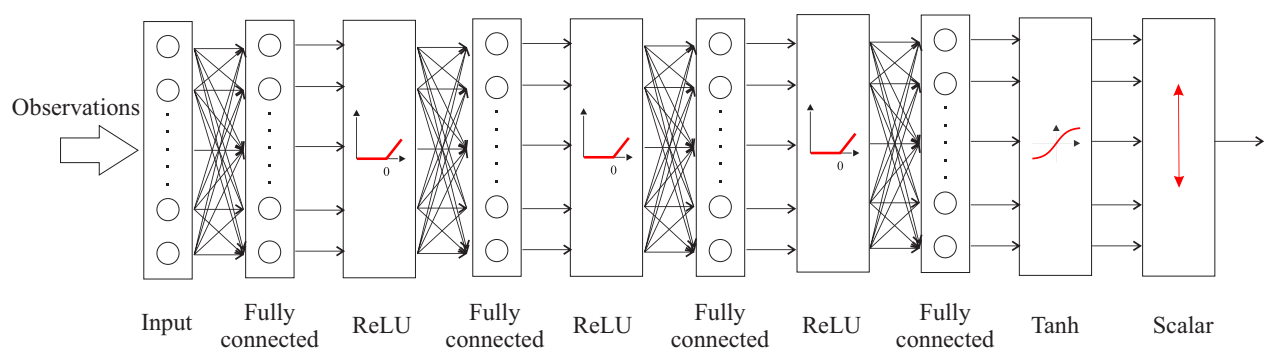

Figure 7: The employed structure for the NN controllers.

\begin{tabular}{cccccc||cccc}
\multicolumn{1}{c}{$\mathrm{NN}$ (30 Neurons per Layer) } \\
\hline$c$ & $\alpha_{d}$ & $\delta_{n, m}$ & Samples & $T(s)$ & $\mathcal{A}$ & $\delta_{n, m}$ & Samples & $T(s)$ & $\mathcal{A}$ \\
\hline 0.40 & 0.99 & 0.98 & $4.3 \mathrm{e}+01$ & $7.4 \mathrm{e}-03$ & $\mathrm{~F}$ & 0.36 & $1.0 \mathrm{e}+04$ & $9.6 \mathrm{e}+00$ & $\mathrm{~T}$ \\
0.40 & 0.95 & 1.00 & $1.9 \mathrm{e}+01$ & $3.1 \mathrm{e}-03$ & $\mathrm{~F}$ & 0.36 & $3.6 \mathrm{e}+03$ & $2.0 \mathrm{e}+00$ & $\mathrm{~T}$ \\
0.25 & 0.99 & 1.00 & $2.5 \mathrm{e}+01$ & $4.1 \mathrm{e}-03$ & $\mathrm{~F}$ & 0.37 & $9.5 \mathrm{e}+02$ & $3.2 \mathrm{e}-01$ & $\mathrm{~F}$ \\
0.25 & 0.95 & 1.00 & $1.3 \mathrm{e}+01$ & $2.1 \mathrm{e}-03$ & $\mathrm{~F}$ & 0.42 & $2.5 \mathrm{e}+02$ & $5.9 \mathrm{e}-02$ & $\mathrm{~F}$ \\
0.10 & 0.99 & 1.00 & $1.8 \mathrm{e}+01$ & $3.0 \mathrm{e}-03$ & $\mathrm{~F}$ & 0.36 & $2.1 \mathrm{e}+02$ & $4.2 \mathrm{e}-02$ & $\mathrm{~F}$ \\
0.10 & 0.95 & 1.00 & $9.0 \mathrm{e}+00$ & $1.6 \mathrm{e}-03$ & $\mathrm{~F}$ & 0.35 & $1.2 \mathrm{e}+02$ & $2.2 \mathrm{e}-02$ & $\mathrm{~F}$ \\
0.05 & 0.99 & 1.00 & $1.6 \mathrm{e}+01$ & $2.7 \mathrm{e}-03$ & $\mathrm{~F}$ & 0.38 & $1.3 \mathrm{e}+02$ & $2.5 \mathrm{e}-02$ & $\mathrm{~F}$ \\
0.05 & 0.95 & 1.00 & $8.0 \mathrm{e}+00$ & $1.2 \mathrm{e}-03$ & $\mathrm{~F}$ & 0.36 & $7.3 \mathrm{e}+01$ & $1.4 \mathrm{e}-02$ & $\mathrm{~F}$ \\
\hline
\end{tabular}

Table 2: Statistical verification results for the conformance property (26) and the test statistics $\delta_{n, m}$ upon Algorithm 2 termination for different values of the conformance parameter $c$ and desired confidence level $\alpha_{d}$.

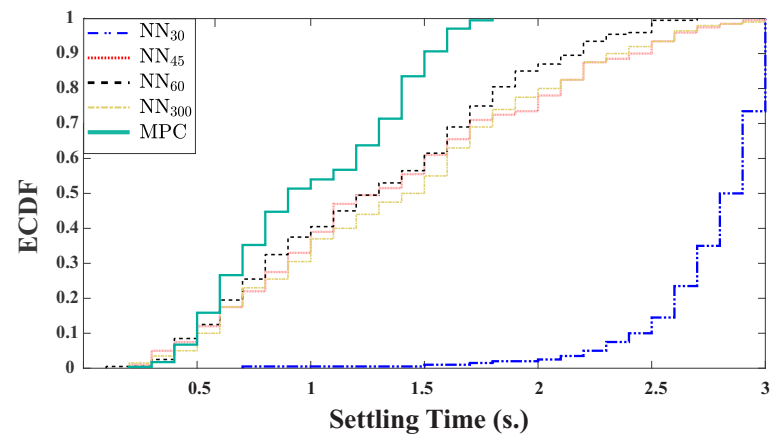

Figure 8: The ECDFs of the settling time for the MPC and NN-based controllers with with $30,45,60$, and 3000 neurons per layer $\left(\mathbf{N N}_{30}, \mathbf{N N}_{45}, \mathbf{N N}_{60}\right.$, and $\left.\mathbf{N N}_{300}\right)$ from 200 samples. The conformance is visually demonstrated by the closeness of the ECDFs.

beyond 45 does not lead to considerable change in the CDF of the settling times for $\mathrm{NN}$ based controllers. Comparing to $\mathrm{NN}_{300}$, the $\mathrm{NN}_{60}$ controller has better conformance with the MPC. The latter implies that $\mathrm{NN}_{300}$ controller has the over-fitting problem. For the NN-based controllers of different sizes, the test statistics upon algorithm termination is $\delta_{n, m}^{\mathrm{NN}_{30}}=0.98, \delta_{n, m}^{\mathrm{NN}_{45}}=0.31, \delta_{n, m}^{\mathrm{NN}_{60}}=0.31$, and $\delta_{n, m}^{\mathrm{NN}_{300}}=0.35$.

\section{RELATED WORK}

Conformance of CPS for different types of specifications of interest is studied in [13, 18, 21, 23, 32, 34]. As in [1, 9], in this work, we focus on a class of conformance properties for CPS that are specified by temporal logic formulas. Our notion of conformance can be viewed as the probabilistic extension of $[1,9]$, that is needed to allow for capturing the conformance between a wide class of probabilistic CPS (which we model as PUSs). Since reachability properties can be in general captured by temporal logic formulas, our notion of conformance is more general than the conformance for reachability from [21, 32].

Existing works on conformance for temporal logic specifications mainly focus on non-probabilistic models $[1,9,13,18,21]$. On the other hand, in this work, we focus on a probabilistic notion of conformance - the satisfaction probability of the specifications of interest should be approximately equal. In [1, 9], conformance builds a relation between two models such that if any STL formula holds on one model, then the corresponding formula should automatically hold on the other model. Conceptually, our notion of conformance is less stringent, as it only involves a given set of STL formulas of interest. Furthermore, our notion of conformance is conceptually more general than [21,32], where the conformance is only for reachability. Our notion of conformance can specify the conformance of probabilistic reachability for two models.

Conformance is different from the simulation/bisimulation [7] in two aspects. Conceptually, conformance focuses on the level of functionality, and only captures the similarity between two models for a set of specifications of interest. That is, the behavior of the two models may be very different for other specifications (not of interest). On the other hand, the simulation/bisimulation focuses on the level of executions, and requires an execution-wise correspondence between the two models. Also, the two concepts have slightly different domains of applications $[1,9,18]$. Conformance is commonly only used for cyber-physical and embedded control systems, while 
simulation/bisimulation may be used for both discrete models [7] as well as cyber-physical and embedded control systems [17, 39].

To the best of our knowledge, this is the first work on statistically verifying the probabilistic conformance of CPS with complex dynamics (formally captured as probabilistic uncertain systems from Definition 1), while providing provable confidence levels (i.e., false positive/negative ratios). Existing model-based methods for conformance, such as $[1,9,18,21]$ cannot directly handle such systems with complex or even unknown dynamics in practice. On the other hand, existing conformance testing methods for temporal logic specifications $[13,32]$ or other specifications $[23,34]$ cannot provide probabilistic guarantees like the presented method. Therefore, those methods are not directly comparable with ours for the case studies presented in Section 6.

\section{CONCLUSION}

In this paper, we proposed a new concept of probabilistic conformance for CPS. This notion is based on approximately equal satisfaction probabilities for a given (infinite) set of signal temporal logic (STL) formulas. We introduced a verification algorithm for the probabilistic conformance of grey-box CPS, modeled by probabilistic uncertain systems. Our statistical verification algorithm is based on a new statistical test that can check if two probability distributions are equal for any desired confidence level (lower than 1). Finally, we used our approach to verify (1) the nonconformity in the startup time of the full and simplified models of the Toyota powertrain system, (2) the approximate conformity in the settling time of the model predictive control (MPC) based lane-keeping controller and neural network (NN)-based lane-keeping controllers of sufficient sizes, and (3) the nonconformity in the maximal DC voltage deviation of the full and simplified model of a power grid system. An avenue for future work is to support conformance verification of systems for security/privacy policies that are hyperproperties. Besides, there is a need to go beyond verification and develop techniques to identify system behaviors that result in nonconformity.

\section{ACKNOWLEDGMENTS}

This work is sponsored in part by the ONR under agreements N00014-17-1-2504 and N00014-20-1-2745, AFOSR under award number FA9550-19-1-0169, as well as the NSF CNS-1652544 and SaTC1813388 awards.

\section{REFERENCES}

[1] Houssam Abbas, Hans Mittelmann, and Georgios Fainekos. 2014. Formal Property Verification in a Conformance Testing Framework. In 12th ACM/IEEE Conf. on Formal Methods and Models for Codesign (MEMOCODE). 155-164.

[2] Gul Agha and Karl Palmskog. 2018. A Survey of Statistical Model Checking. ACM Trans. Model. Comput. Simul. 28, 1 (2018), 6:1-6:39.

[3] Eugene Asarin, Alexandre Donzé, Oded Maler, and Dejan Nickovic. 2011. Parametric identification of temporal properties. In International Conference on Runtime Verification. 147-160.

[4] Steven RH Barrett, Raymond L Speth, Sebastian D Eastham, Irene C Dedoussi, Akshay Ashok, Robert Malina, and David W Keith. 2015. Impact of the Volkswagen emissions control defeat device on US public health. Environmental Research Letters 10, 11 (2015), 114005.

[5] Gilles Barthe, Pedro R D’Argenio, Bernd Finkbeiner, and Holger Hermanns. 2016 Facets of software doping. In International Symposium on Leveraging Applications of Formal Methods. Springer, 601-608.

[6] CPSL@Duke. 2020. Probabilistic Conformance for CPS: Case-Studies. https: //gitlab.oit.duke.edu/cpsl/conformance.
[7] Dennis Dams and Orna Grumberg. 2018. Abstraction and Abstraction Refinement. In Handbook of Model Checking. Springer International, 385-419.

[8] Moacyr A. G. De Brito, Leonardo P. Sampaio, G. Luigi, Guilherme A. e Melo, and Carlos A. Canesin. 2011. Comparative analysis of MPPT techniques for PV applications. In 2011 International Conference on Clean Electrical Power. 99-104.

[9] Jyotirmoy V. Deshmukh, Rupak Majumdar, and Vinayak S. Prabhu. 2017. Quantifying Conformance Using the Skorokhod Metric. Formal Methods in System Design 50, 2 (2017), 168-206.

[10] Jyotirmoy V. Deshpande, Uttara Naik-Nimbalkar, and Isha Dewan. 2018. Nonparametric Statistics: Theory and Methods.

[11] Giovanni Fasano and Alberto Franceschini. 1987. A multidimensional version of the Kolmogorov-Smirnov test. Monthly Notices of the Royal Astronomical Society 225, 1 (1987), 155-170.

[12] Thomas D Gillespie. 1992. Fundamentals of vehicle dynamics. Vol. 400. Society of automotive engineers Warrendale, $\mathrm{PA}$

[13] Alexander Graf-Brill and Holger Hermanns. 2019. Component-aware InputOutput Conformance. In International Conference on Formal Techniques for Distributed Objects, Components, and Systems. 111-128.

[14] Lex Heerink and Jan Tretmans. 1996. Formal Methods in Conformance Testing: A Probabilistic Refinement. In Int. Work. on Testing of Communicating Sys. 261-276.

[15] Thomas A Henzinger. 2000. The theory of hybrid automata. In Verification of Digital and Hybrid Systems. Springer, 265-292.

[16] Xiaoqing Jin, Jyotirmoy V. Deshmukh, James Kapinski, Koichi Ueda, and Ken Butts. 2014. Powertrain Control Verification Benchmark. In The 17th International Conference on Hybrid Systems: Computation and Control. 253-262.

[17] Augung A. Julius and George J. Pappas. 2009. Approximations of Stochastic Hybrid Systems. IEEE Trans. Automat. Control 54, 6 (2009), 1193-1203.

[18] Narges Khakpour and Mohammad Reza Mousavi. 2015. Notions of Conformance Testing for Cyber-Physical Systems: Overview and Roadmap. In 26th International Conference on Concurrency Theory (CONCUR), Vol. 42. 18-40.

[19] Kim G. Larsen and Axel Legay. 2016. Statistical Model Checking: Past, Present, and Future. In Leveraging Applications of Formal Methods, Verification and Validation: Foundational Techniques. 3-15.

[20] Axel Legay, Benoît Delahaye, and Saddek Bensalem. 2010. Statistical Model Checking: An Overview. In Runtime Verification. Vol. 6418. 122-135.

[21] Stephan B. Liu and Matthias Althoff. 2018. Reachset Conformance of Forward Dynamic Models for the Formal Analysis of Robots. In IEEE/RSF International Conf. on Intelligent Robots and Systems (IROS). 370-376.

[22] Natalia López, Manuel Núñez, and Ismael Rodríguez. 2006. Specification, Testing and Implementation Relations for Symbolic-Probabilistic Systems. Theoretical Computer Science 353, 1-3 (2006), 228-248.

[23] Rupak Majumdar, Indranil Saha, Koichi Ueda, and Hakan Yazarel. 2013. Compositional equivalence checking for models and code of control systems. In 52nd IEEE Conference on Decision and Control. 1564-1571.

[24] Oded Maler and Dejan Nickovic. 2004. Monitoring temporal properties of continuous signals. In Formal Techniques, Modelling and Analysis of Timed and Fault-Tolerant Systems. Springer, 152-166.

[25] MathWorks, Inc. 2019. lane-keeping Assist System,. https://www.mathwork $\mathrm{s.com} / \mathrm{help} / \mathrm{mpc} / \mathrm{ug} / \mathrm{lane}$-keeping-assist-system-using-model-predictivecontrol.htmll. Accessed: 2019-7-15.

[26] MathWorks, Inc. 2019. Model Predictive Control Toolbox. https://www.math works.com/help/mpc/ug/lane-keeping-assist-system-using-model-predictivecontrol.html. Accessed: 2019-7-15.

[27] MathWorks, Inc. 2019. PowerSim. https://www.mathworks.com/help/physmod /sps/index.html?s_tid=CRUX_lftnav. Accessed: 2019-7-15.

[28] MathWorks, Inc. 2019. SimPower. https://www.mathworks.com/help/physmod/s ps/examples/250-kw-grid-connected-pv-array.html. Accessed: 2019-7-15.

[29] Yunpeng Pan, Ching-An Cheng, Kamil Saigol, Keuntaek Lee, Xinyan Yan, Evangelos Theodorou, and Byron Boots. 2017. Agile autonomous driving using end-to-end deep imitation learning. arXiv preprint arXiv:1709.07174 (2017).

[30] John A. Peacock. 1983. Two-dimensional goodness-of-fit testing in astronomy. Monthly Notices of the Royal Astronomical Society 202, 3 (1983), 615-627.

[31] Srinivas Pinisetty, Gerardo Schneider, and David Sands. 2018. Runtime Verification of Hyperproperties for Deterministic Programs. In Proceedings of the 6th Conference on Formal Methods in Software Engineering - FormaliSE '18. 20-29.

[32] Hendrik Roehm, Jens Oehlerking, Matthias Woehrle, and Matthias Althoff. 2016. Reachset Conformance Testing of Hybrid Automata. In 19th International Conference on Hybrid Systems: Computation and Control (HSCC). 277-286.

[33] Nima Roohi, Yu Wang, Matthew West, Geir E. Dullerud, and Mahesh Viswanathan. 2017. Statistical Verification of the Toyota Powertrain Control Verification Benchmark. In Proceedings of the 20th International Conference on Hybrid Systems: Computation and Control (HSCC). 65-70.

[34] Michael Ryabtsev and Ofer Strichman. 2009. Translation validation: From simulink to c. In International Conference on Computer Aided Verification. 696-701.

[35] Jeremy Sproston. 2000. Decidable Model Checking of Probabilistic Hybrid Automata. In Formal Techniques in Real-Time and Fault-Tolerant Systems. 31-45.

[36] Kishor S. Trivedi, Andrea Bobbio, and Jogesh Muppala. 2017. Reliability and Availability Engineering: Modeling, Analysis, and Applications. 
[37] A. W. van der Vaart and Jon A Wellner. 1996. Glivenko-Cantelli Theorems. In Weak Convergence and Empirical Processes. Springer, 122-126.

[38] Yu Wang, Siddhartha Nalluri, Borzoo Bonakdarpour, and Miroslav Pajic. 2021 Statistical model checking for hyperproperties. In IEEE Computer Security Foundations Symposium (CSF). To appear.

[39] Yu Wang, Nima Roohi, Matthew West, Mahesh Viswanathan, and Geir E. Dullerud. 2015. Statistical Verification of Dynamical Systems Using Set Oriented Methods. In 18th Int. Conf. on Hybrid Systems: Computation and Control (HSCC). 169-178.

[40] Yu Wang, Mojtaba Zarei, Borzoo Bonakdarpour, and Miroslav Pajic. 2019. Statistical Verification of Hyperproperties for Cyber-Physical Systems. ACM Transactions on Embedded Computing Systems 18, 5s (2019), 1-23.

[41] Mojtaba Zarei, Yu Wang, and Miroslav Pajic. 2020. Statistical verification of learning-based cyber-physical systems. In Proceedings of the 23rd International Conference on Hybrid Systems: Computation and Control (HSCC). 1-7.

[42] Lijun Zhang, Zhikun She, Stefan Ratschan, Holger Hermanns, and Ernst Moritz Hahn. 2010. Safety Verification for Probabilistic Hybrid Systems. In Computer Aided Verification. 196-211.

\section{A APPENDIX}

\section{A.1 Power Plant Case-Study}

In the final case-study, we compare the detailed and average models of a $100 \mathrm{~kW}$ array connected to a $25 \mathrm{kV}$ grid via a DC-DC boost converter and a three-phase three-level Voltage Source Converter (VSC), from the MATLAB Simscape Electrical Toolbox [27] Both models include a Photo Voltaic (PV) array that delivers the maximum power of $100 \mathrm{~kW}$ at $1000 \mathrm{~W} / \mathrm{m}^{2}$ sun irradiance, a DC-DC boost converter, 3-level 3-phase VSC, capacitor bank, three-phase coupling transformer, and a given utility grid. The models use the Simulink model of a boost converter to implement the Maximum Power Point Tracking (MPPT). The MPPT optimizes the match between the solar array (PV panels) and the utility grid. The models have differences such as employed technique to implement MPPT, DC-DC, and VSC converters' structure [8].

The VSC converts the $500 \mathrm{~V}$ DC link voltage to $260 \mathrm{~V} \mathrm{AC}$ and keeps unity power factor. To this end, two control loops are employed: one control loop regulates DC link voltage to $\pm 250 \mathrm{~V}$ (external controller) and the other control loop regulates active and reactive grid currents (internal controller). The active current reference is the output of the DC voltage external controller. The latter controller is a PI (proportional-integral) controller whose input is the error of the DC voltage.

Conformance. We consider the deviation of the DC voltage $e_{v d c}$, when the sun irradiance and environment temperature are subject to changes. For an arbitrary threshold $\gamma$, we use the STL specification $\square_{[0.5,2]}\left(\left|e_{v d c}\right|<\gamma\right)$, which is monotonically parametrized by $\gamma$, to capture that $e_{v d c}$ is always below $\gamma$ within the time interval $[0.5,2]$ of interest. Accordingly, the conformance between the detailed and average models for this parametrized specification is captured by

$$
\begin{aligned}
\forall \gamma \geq 0 . & \operatorname{Pr}_{\sigma_{d} \sim \mathcal{M}_{d}}\left(\sigma_{d} \mid=\square_{[0.5,2]}\left(\left|e_{v d c_{d}}\right|<\gamma\right)\right) \\
& \approx_{c} \operatorname{Pr}_{\sigma_{a} \sim \mathcal{M}_{a}}\left(\sigma_{a} \models \square_{[0.5,2]}\left(\left|e_{v d c_{a}}\right|<\gamma\right)\right),
\end{aligned}
$$

where the constant $c>0$, the approximate equality $\approx_{c}$ means the difference is less than $c$, and the detailed and average models are denoted by $d$ and $a$, respectively.

We applied Algorithm 2 with parameters $\alpha \in\{0.95,0.99\}$ and $c \in\{0.001,0.005,0.01,0.05\}$. For both the models, we consider the standard test conditions (initial temperature and irradiance are $25^{\circ}$ and $1000 \mathrm{~W} / \mathrm{m}^{2}$, respectively) with the following scenario (i.e., the input to the models):

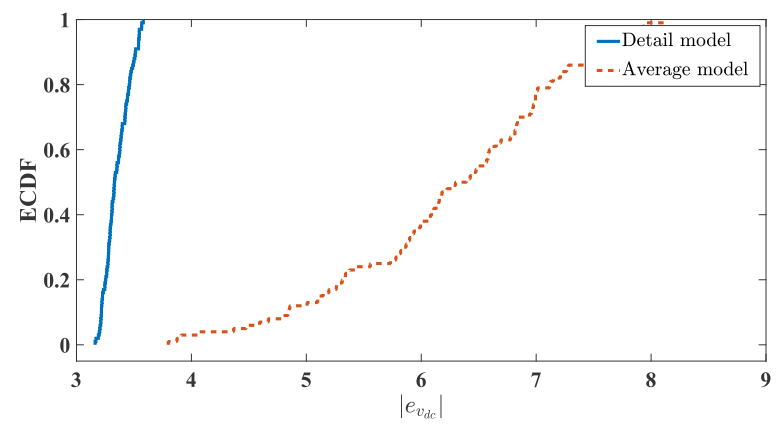

Figure 9: The ECDFs for the maximum deviation of $V_{d c}$ in the detailed and average models for 100 samples. The two distributions of the maximum errors for the models are noticeably different.

\begin{tabular}{llllll}
$c$ & $\alpha_{d}$ & $\delta_{n, m}$ & Samples & Time $(\mathrm{sec})$. & $\mathcal{A}$ \\
\hline 0.40 & 0.99 & 1.00 & $3.9 \mathrm{e}+01$ & $1.0 \mathrm{e}-02$ & $\mathrm{~F}$ \\
0.40 & 0.95 & 1.00 & $1.9 \mathrm{e}+01$ & $6.9 \mathrm{e}-03$ & $\mathrm{~F}$ \\
0.25 & 0.99 & 1.00 & $2.5 \mathrm{e}+01$ & $5.3 \mathrm{e}-03$ & $\mathrm{~F}$ \\
0.25 & 0.95 & 1.00 & $1.3 \mathrm{e}+01$ & $3.3 \mathrm{e}-03$ & $\mathrm{~F}$ \\
0.10 & 0.99 & 1.00 & $1.8 \mathrm{e}+01$ & $3.8 \mathrm{e}-03$ & $\mathrm{~F}$ \\
0.10 & 0.95 & 1.00 & $9.0 \mathrm{e}+00$ & $1.8 \mathrm{e}-03$ & $\mathrm{~F}$ \\
0.05 & 0.99 & 0.94 & $1.8 \mathrm{e}+01$ & $3.2 \mathrm{e}-03$ & $\mathrm{~F}$ \\
0.05 & 0.95 & 1.00 & $8.0 \mathrm{e}+00$ & $1.3 \mathrm{e}-03$ & $\mathrm{~F}$ \\
\hline
\end{tabular}

Table 3: Statistical verification results of the conformance property (27) and the test statistics $\delta_{n, m}$ upon Algorithm 2 termination, for different conformance parameter $c$ and desired confidence level $\alpha_{d}$.

(1) At $t=0.3 \mathrm{~s}$ MPPT starts to regulate PV voltage.

(2) In time interval $[0.6,1.1] s$, the sun irradiance linearly is ramped to a minimum value. Also, the environment temperature start increasing to a maximum value, simultaneously.

(3) In time interval $[1.1,1.2] \mathrm{s}$, the sun irradiance and environment temperature stay constant. The minimum value of the irradiance is drawn randomly from a distribution $\mathrm{N}_{\text {ir }}\left(650,10^{2}\right)$ and the maximum temperature is $20-0.02 \times$ $\mathrm{N}_{\text {ir }}\left(650,10^{2}\right)$.

(4) In time interval $[1.2,1.7] \mathrm{s}$, the sun irradiance and temperature are linearly restored back to $1000 \mathrm{~W} / \mathrm{m}^{2}$ and $25^{\circ}$, respectively; from then onward, remain constant.

Result Analysis. Table 3 contains the results that demonstrate the nonconformance of the detailed and average models for the requirement (27), although it is commonly believed that the average model is generally a good approximation of the detailed model [27]. This result is achieved with a relatively small number of samples (at most a few dozen samples for each setup). The results for the considered specification reveals that two models do not have conformance for any values of $c$. To confirm the results of Table 3, we plot in Figure 9 the ECDFs of the maximum deviation $\left|e_{V_{d c}}\right|$ of the detailed and average models; the discrepancy of the two ECDFs 
demonstrates the nonconformance of two models for the requirement (27). Each ECDF uses 100 samples, which is significantly more than the samples required by Algorithm 2, as shown by Table 3. 